\title{
CORRECTION
}

View Article Online

View Journal I View Issue

\section{D) Check for updates \\ Correction: A continuum membrane model can predict curvature sensing by helix insertion}

Cite this: Soft Matter, 2022,

18,683

Yiben Fu, ${ }^{a}$ Wade F. Zeno, ${ }^{\text {b Jeanne C. Stachowiak }}{ }^{\mathrm{c}}$ and Margaret E. Johnson ${ }^{\star a}$

Correction for 'A continuum membrane model can predict curvature sensing by helix insertion' by Yiben

DOI: $10.1039 / \mathrm{d} 1 \mathrm{sm} 90225 \mathrm{c}$

Fu et al., Soft Matter, 2021, 17, 10649-10663, DOI: 10.1039/D1SM01333E.

rsc.li/soft-matter-journal

The authors regret the omission of some funders from the acknowledgements section in the original article. The Acknowledgements section should read as follows:

MEJ gratefully acknowledges funding from an NIH MIRA R35GM133644, and an NSF CAREER Award 1753174. JCS was supported by R35GM139531 from the National Institutes of Health and by the Welch Foundation F-2047. We acknowledge supercomputing resources provided by ARCH at Johns Hopkins University, and the NSF-MRI funded (Award 1920103) rockfish cluster. We thank Dr Alexander Sodt and Prof Brian Camley for helpful discussions. We also thank Prof Tobias Baumgart for kindly sharing data.

The Royal Society of Chemistry apologises for these errors and any consequent inconvenience to authors and readers.

\footnotetext{
${ }^{a}$ T. C. Jenkins Department of Biophysics, The Johns Hopkins University, 3400 N. Charles St., Baltimore, Maryland 21218, USA. E-mail: margaret.johnson@jhu.edu

${ }^{b}$ Mork Family Department of Chemical Engineering and Materials Science, The University of Southern California, Los Angeles, California, 90089, USA

${ }^{c}$ Department of Biomedical Engineering, The University of Texas at Austin, Austin, Texas 78712, USA
} 